und $y$ ausdrücken. Das heißt also, daß wieder $a, b, a^{\prime}$ und $b^{\prime}$ Funktionen $\operatorname{der} 9$ voneinander unabhängigen Größen $D, E, F$, $\mathrm{d} D / \mathrm{d} x, \mathrm{~d} E / \mathrm{d} x, \mathrm{~d} F / \mathrm{d} x, \mathrm{~d} D / \mathrm{d} y, \mathrm{~d} E / \mathrm{d} y$ und $\mathrm{d} F / \mathrm{d} y$ sind. Zwischen diesen 9 Größen bestehen aber zwei Abhängigkeiten, und die 7 restierenden voneinander unabhängigen Größen bestimmen gemeinsam die Krümmungsverhältnisse in dem Punkte und in seiner nächsten Umgebung.

Jede geodätische Verbindung zwischen zwei Punkten, in welchen astronomische Messungen von Länge, Breite und Azimut vorgenommen sind, gibt, wie wir gesehen haben, drei Gleichungen zwischen $a, b, a^{\prime}$ und $b^{\prime}$, also drei Gleichungen zwischen -den besprochenen 7 charakteristischen Größen.

Wird ein Punkt mit drei benachbarten Punkten verbunden, so erhält man 9 Gleichungen zur Bestimmung der 7 Größen.

Ein Netz von der Form a gibt also eine vollständige Bestimmung mit zwei Überbestimmungen für sämtliche Netzpunkte. Hat das Netz die Form b, so erhält man für jeden Punkt fünf Überbestimmungen. Ein Netz (c), wie das französische, würde, wenn sich in sämtlichen Knotenpunkten asitronomische Messungen vorfänden, ebenso für jeden Punkt fünf Uberbestimmungen geben. Wäre dagegen das Netz aus Dreiecken (wie d) gebildet, so würde jeder Netzpunkt I I Überbestimmungen geben.

Wenn aber die Verhältnisse solche sind, daß man nichts Bestimmtes auf $A_{0}$ bauen darf, dann sind für die Bestimmung der 7
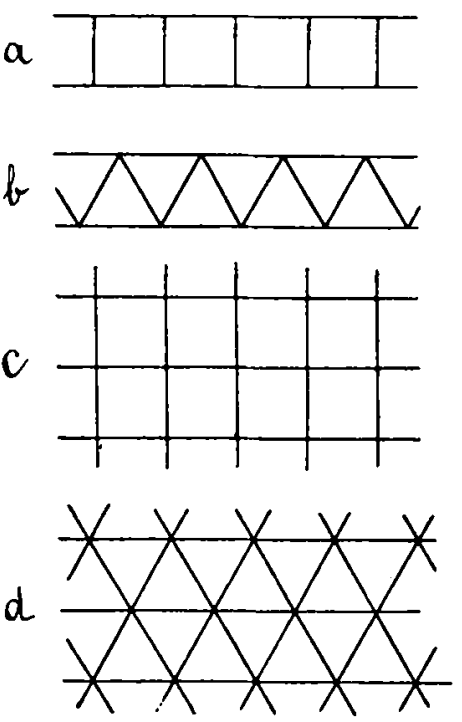

Figur 2.
Größen vier Verbindungen nötig (welche doch eine Überbestimmung geben), und von den vier genannten Netzformen geben nur die drei letzten eine völlige Bestimmung mit resp. $I$, 1 und 5 Überbestimmungen für jeden Punkt.

Wie $\boldsymbol{A}$ ausgenützt werden soll, hängt natürlich davon ab, wie nahe man daran ist, die Größe $\Lambda_{0}$ mit einer für die Praxis genügenden Sicherheit $=0$ setzen zu können. Jedoch kommt es mir vor, daß es immer eine große Gefahr in sich schließen muß, sich auf die Gleichung $\Lambda_{0}=0$ zu verlassen, denn die Bestimmung von $m^{\prime}$ kann leider nie sicher sein, und das besprochene Beispiel zeigt ja, wie außerordentlich groß der Einfluß dieser Größe ist.

Zum Schluß soll noch einmal hervorgehoben werden, daß die negative Seite dieser Abhandlung die Hauptsache ist. Es ist danach zweifellos, daß die Verhältnisse so sein können, daß das Theorem von Laplace unanwendbar ist. Der Beweis für seine Anwendbarkeit, muß in jedem einzelnen Fall von der Praxis geliefert werden, und wenn auch die Praxis die Anwendbarkeit des 'Theorems bisweilen $z u$ bestätigen scheint, so ist doch hierdurch kein Beweis für seine Korrektheit geliefert, sondern nur eine Eigenschaft der Fläche zu Tage getreten.

Die Schlußfehler der Laplaceschen Gleichungen in Mitteleuropa scheinen z. B. den Beweis dafür zu liefern, daß die Lotabweichungen hier zum Teil so unregelmäßig variieren müssen, daß sie mehr als Zufälligkeiten als wie als systematische Variationen auftreten, wodurch die Reihenentwickelungen wertlos werden.

Überhaupt kann schon das für die Anwendbarkeit gegebene Kriterium nicht nur durch den Linienzug, sondern auch durch die Beschaffenheit der Fläche unbrauchbar gemacht werden, und das ist noch mehr der Fall mit dem hier zur Bestimmung der Flächenelemente gemachten Versuch, denn alles dies beruht auf der doppelten Voraussetzung: die Möglichkeit, die Dreiecksreihe durch eine stetige Kurve und das Geoid durch eine glattere Fläche ersetzen zu können.

Hellerup, I 9 I 3 Dezember.

F. A. Buchwaldt.

\title{
Real Paths of fireballs and shooting stars observed in England 1912 and 1913.
} By $W . F$. Denning.

The following table of the heights, radiants etc, of meteors is continued from a list in the Monthly Notices of the R. A.S. for I9 2 March in which I gave the real paths of 429 meteors observed in the years from 1897 to $191 \mathrm{I}$ inclusive. I believe that the details given may be regarded with confidence as the observations were conducted with a special view to the computation of the real paths and the persons engaged were extremely careful in obtaining the records. It is thought that the accumulation of data of this character will be valuable not only in corroborating the positions of the major and minor showers but will also be important for determining the average heights and velocities of meteors and in dividing them into classes according to their different characteristics.

Mrs. Fiammetta Wilson has worked in this field with remarkable perseverance and enthusiasm and to her successful efforts the interesting, and pretty abundant nature of the results are mainly due.

\begin{tabular}{|c|c|c|c|c|c|c|c|c|c|c|c|}
\hline 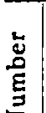 & Date & & Gr. M. T. & 总 & 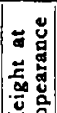 & 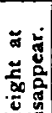 & 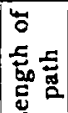 & 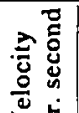 & & & 8 \\
\hline & & & & & & & & & $\alpha$ & 8 & \\
\hline & 1912 & & & & mil. & mil. & miles & miles & & & \\
\hline $\mathbf{I}$ & Mar. & 6 & $8^{h} \quad 4^{m}$ & $>D$ & 72 & 34 & 295 & I 7 & $17^{\circ}$ & $+6^{\circ}$ & 11 \\
\hline 2 & & 9 & 850 & $2 \times \%$ & 67 & 67 & 70 & 20 & 18 & +9 & \\
\hline 3 & & 6 & 9 IO & $>$ \% & 55 & 43 & 133 & 22 & I 74 & -27 & \\
\hline & & & & $>D$ & 62 & 39 & $2 \begin{array}{lll}2 & 10\end{array}$ & 28 & 208 & -10 & \\
\hline 4 & & 8 & 245 & $>D$ & 76 & 26 & 120 & 2 I & 2 & -2 & \\
\hline 5 & April & 8 & 9 I 5. & $>q$ & 56 & 40 & 87 & 22 & 210 & -8 & \\
\hline 6. & & 0 & I 433 & $>D$ & 1 & 49 & 130 & 10 & 195 & -30 & \\
\hline 7 & June I & & I I I 4 & 24 & 9 & 29 & 30 & 10 & 270 & +47 & \\
\hline 8 & July & 4 & I I 5 & $q$ & 68 & 49 & 2 I & 26 & 300 & +25 & \\
\hline 9 & & & 1030 & 3 & 69 & 55 & I 9 & 24 & 60 & +87 & \\
\hline 10 & Aug. I & & IO 48 & I & 94 & $5^{1}$ & 80 & 47 & 46 & +59 & \\
\hline I I & Sept. & 5 & 730 & \& & 78 & 44 & 38 & 19 & 235 & +51 & \\
\hline I2! & Oct. & 2 & 840 & $\mathbf{I}$ & 64 & 31 & 37 & I 5 & 349 & +39 & \\
\hline
\end{tabular}




\begin{tabular}{|c|c|c|c|c|c|c|c|c|c|c|c|c|c|c|c|c|c|c|c|c|c|c|c|}
\hline 䓌 & Date & & Gr. M. T. & 总 & 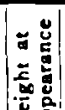 & 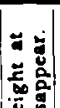 & 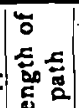 & 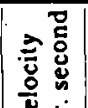 & & & : & E & Date & & Gr. M. T. & 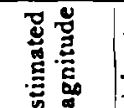 & 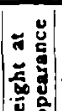 & 艾 & 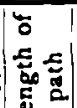 & 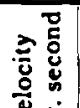 & & & 5 \\
\hline & & & & & & & & & $\alpha$ & 8 & & & & & & & & & & & $a$ & 0 & \\
\hline & 2 & & & & mil. & mil. & miles & miles & & & & & 1913 & & & & mil. & mil. & miles & miles & & & \\
\hline 13 & Oct. & 8 & $8^{h} 3^{m}$ & $>1$ & 65 & 27 & 57 & 23 & $214^{\circ}$ & $+64^{\circ}$ & 3 & 20 & Aug. 2 & 22 & $9^{h} \times 9^{m}$ & 2 & 48 & 48 & 160 & 10 & $208^{\circ}$ & $-7^{\circ}$ & \\
\hline 14 & & 8 & $901 / 2$ & $>1$ & 81 & 48 & 78 & 18 & 32 & +8 & 3 & 21 & & 251 & 1022 & 4 & 75 & 51 & 24 & 36 & $3 \times 4$ & +45 & 3 \\
\hline 15 & Dec. & $\mathbf{I} \mathbf{I}$ & $103^{61 / 2}$ & $I-24$ & 71 & 43 & $3^{8}$ & 16 & 53 & +25 & 3 & 22 & & & 1041 & $\mathbf{I}$ & 69 & 62 & 16 & 30 & 348 & +2 & \\
\hline 16 & & 12 & 87 & $>I$ & 76 & 49 & 77 & 24 & I 18 & +32 & 2 & 23 & & & 1046 & 3 & 64 & 54 & 18 & 18 & 348 & +2 & 3 \\
\hline & 1913 & & & & & & & & & & & 24 & & & II & 4 & 64 & $5 \mathrm{I}$ & 29 & 20 & 200 & +55 & 3 \\
\hline $\mathbf{I}$ & Jan. & I & 721 & $>I$ & 71 & 55 & 45 & 18 & 134 & +33 & 2 & 25 & Sept. & 61 & 1014 & $4^{-I}$ & 72 & 48 & 25 & - & 304 & +40 & 3 \\
\hline 2 & & 2 & 735 & $>9$ & 85 & 42 & 55 & I 5 & 75 & +30 & 2 & 26 & & 8 & 944 & $3^{-2}$ & 24 & 15 & 17 & 17 & 321 & -9 & 3 \\
\hline 3 & & 9 I & IO $29^{1} / 2$ & $>I$ & 72 & 45 & 44 & I 9 & 353 & +52 & 3 & 27 & & & 954 & $4-3$ & 80 & 62 & 40 & 20 & 21 & +14 & 3 \\
\hline 4 & Febr. & 8 & 834 & $>I$ & 67 & 43 & 47 & $10^{i} / 2$ & 32 & +9 & 2 & 28 & & & 1010 & $3^{-2}$ & 80 & 58 & $3^{8}$ & 30 & 44 & +37 & 4 \\
\hline 5 & & 9 & $\begin{array}{ll}6 & 43\end{array}$ & $2 \times 9$ & 48 & 16 & 32 & 一 & 47 & +46 & 4 & 29 & & IIII & 10.16 & $>D$ & $8 I$ & 66 & 44 & & 74 & +41 & ) \\
\hline 6 & Apr & 22 & $10 \quad 221 / 4$ & $2-1$ & 79 & 61 & 36 & - & 271 & +33 & 2 & 29 & & 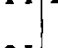 & & & 71 & & & & 76 & +32 & \\
\hline 7 & & 231 & 10 $93 / 4$ & $5^{-2}$ & 46 & 46 & 32 & 32 & 260 & -12 & 2 & 30 & & $2 \mathrm{I}$ & 810 & $\begin{array}{l}2-1 \\
-21\end{array}$ & 84 & 51 & 76 & 38 & 44 & +40 & 3 \\
\hline 8 & May & 24 & I I $24 \%$ & $4-3$ & 64 & 44 & 24 & 19 & $23^{\circ}$ & +17 & 3 & 35 & Oct. & 11 & $10 \quad 21$ & $1-24$ & 54 & 48 & I 2 & 18 & 314 & -11 & \\
\hline 9 & Jur & 14 & 8 & $>D$ & 77 & 29 & $5^{8}$ & 22 & 263 & +64 & 59 & 32 & & 7 & 1035 & $1 / 2$ & 74 & 52 & 86 & $5^{8}$ & 105 & $+3^{6}$ & 0 \\
\hline 10 & & & 10 2 & $2 \times 9$ & 54 & 54 & 490 & 26 & 282 & -23 & 18 & $33 !$ & & 16 & $7 \mathrm{It}$ & $1-2$ & 76 & 55 & 320 & 32 & 164 & +34 & 4 \\
\hline I I & & 16 & I0 $48 \%$ & $2-2$ & 52 & 45 & $3^{8}$ & 一 & 50 & $+{ }_{46}^{6}$ & 3 & 34 & & 22 & 940 & 3 & 50 & 37 & 23 & 17 & 44 & +5 & 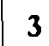 \\
\hline 12 & & 271 & 1136 & 4-3 & 64 & 54 & 20 & 28 & 270 & -6 & 3 & 35 & & 27 & 823 & $x-24$ & 52 & 42 & 59 & 19 & 35 & -11 & 4 \\
\hline I 3 & July & IO I & Io I 7 & $1 / 4 D$ & 66 & 46 & 74 & - & 254 & -22 & 3 & 36 & & & 9 II & $3^{-1}$ & $6 I$ & 46 & 44 & 48 & 46 & +4 & 4 \\
\hline 14 & Aug. & 3 & I 125 & $>D$ & 87 & $2 \mathrm{I}$ & 66 & I 5 & 300 & $+5^{2}$ & 7 & 37 & & 28 & $8 \mathrm{II}$ & $3^{-2}$ & 67 & 47 & 24 & $3^{6}$ & 328 & +73 & \\
\hline & & & & & & & & & 302 & +25 & & $3^{8}$ & Nov. & 7 & 826 & 0 & 66 & 47 & 59 & 15 & 240 & +30 & \\
\hline 15 & & 6 & IO I I & $2 \%$ & 74 & 42 & 59 & - & 40 & +57 & 3 & 39 & & & 926 & $3^{-2}$ & $5^{8}$ & $5 \mathrm{I}$ & 49 & 24 & 72 & +11 & \\
\hline 16 & & & I I I I & $4 \frac{1}{2}$ & $5^{8}$ & 44 & 48 & - & 283 & $-2 I$ & 3 & 40 & & 7 & 95 & I & 54 & 26 & 43 & I 8 & 47 & +6 & \\
\hline 17 & & 8 & 1054 & $1-2 \mid$ & $7 \mathrm{I}$ & 59 & $5 I$ & 34 & 70 & $+4 I$ & 3 & 41 & & $\times 8$ & 626 & $3-2$ & 72 & 64 & 40 & 26 & 132 & +47 & \\
\hline 18 & & & 1142 & $>I$ & 80 & 49 & 47 & 33 & 42 & +57 & 3 & 42 & & & 828 & I & 59 & 42 & 17 & I 2 & $35^{\mathrm{I}}$ & $+5^{2}$ & \\
\hline r & & I I & $95^{8}$ & $2-I$ & 27 & $|18|$ & 12 & 6 & 232 & +52 & 2 & 43 & & 24 & 847 & D. & 66 & 20 & 55 & 14 & 273 & +69 & \\
\hline & & Est & - & & & & & & & & & 44 & ${ }_{1} \mathrm{Dec}$ & 4 & 9 & $1^{-1}$ & $|54|$ & $\begin{array}{ll}21 \\
\mid\end{array}$ & 37 & I I & 43 & +29 & \\
\hline
\end{tabular}

The principal observers in 2 years were Mr. S. A. Wilson and Mrs. Fiammetta Wilson, Bexley Heath, 43, Miss A. Grace Cook, Stowmarket, 20, and $W . F$. Denning, Bristol, 20.

Bristol, I 9 I 4 January I 6.

W. F. Denning.

\section{May metèors from Corona.}

In $1876 \mathrm{Mr}$. $R . P$. Greg's summary of meteoric showers placed the radiant point of the April-May Coronids near $\alpha$ Coronae and $I$ found the position at $231^{\circ}+27^{\circ}$ in $x 887$.

In more recent years however the chief shower is from about $246^{\circ}+30^{\circ}$ near $\xi$ Coronae and a few degrees west of $\zeta$ Herculis.

I observed this shower well both in 1903 and 1911 . The meteors are white, swift and usually devoid of streaks or trains. The best period in which to observe them is between May 18 and 26 . As there will be no moonlight Bristol, 19.4 March 4. to interfere with their display in 1914 , it is hoped that they will be reobserved. The shower presents no very special features of abundance or brilliancy but it is noteworthy as occurring at a period of the year when meteoric phenomena are rare.

The shower furnished some large meteors observed at the Royal Observatory Greenwich on May 18, 1866.

I saw it to the best advantage on May 24, I 9 i i when meteors were unusually frequent, for $I$ counted 18 in $11 / 4$ hour before midnight.

$$
\mathrm{V} \text { Geminorum }=\mathrm{BD}+13^{\circ} 1653^{\circ} \text { var. } \mathrm{e}+13^{\circ} 16558^{\mathrm{m}} .4 .
$$

Il Prof. A. Abetti osservando Pomona s'imbattè nella variabile V Geminorum '), e nelle due sere 6 e 7 Febbraio ne confrontó la posizione con

$$
\begin{aligned}
& *_{\mathrm{I}}=\mathrm{BD}+13^{\circ} 165^{2} 9^{\mathrm{m}} \mathrm{O}=\mathrm{AG} \mathrm{LpzI} 28778^{\mathrm{m}} \cdot 7 \\
& *_{2}=\quad+13^{\delta} 16558.4=28828.2
\end{aligned}
$$

ottenendo per la variabile queste coordinate al I9 14.0 :

$$
\begin{aligned}
& \text { da } * \text { I } \quad \alpha=7^{\mathrm{h}} \times 8^{\mathrm{m}} \times 9: 89 \quad \delta=+13^{\circ} 16^{\prime} \quad 1: 0 \\
& \text { "*2 } 7 \times 8 \times 9.58+1316 \times 8.4
\end{aligned}
$$

dove si riscontra una discordanza specialmente notevole in declinazione $\left.{ }^{2}\right)$. Ridotta la $* 2$ da $W_{1} 7^{\mathrm{h}} 459$, ne venne che

3) Hartwig, Kaivlog und Ephemeriden veränderlicher Sterne fur 1914, Nr. 311, größtes Licht Febr. $10,8 \mathrm{~m} .3$.

') Che sparisce applicando il moto proprio da me trovato e per cui si ha: $\alpha=7^{\mathrm{h}} 18^{\mathrm{m}} 19^{8} 89,0=+13^{\circ} .15^{\circ} 59^{\prime \prime} 3$. 